\title{
APLICAÇÃO DO MODELO SWAT COMO FERRAMENTA PARA ANÁLISES HIDROSSEDIMENTOLÓGICAS NA BACIA HIDROGRÁFICA DO RIO MUTUM PARANÁ - RONDÔNIA
}

Janielson da Silva Lima ${ }^{1}$ Dorisvalder Dias Nunes ${ }^{2}$ Tatiane Emílio Checchia ${ }^{3}$

Resumo: O uso de modelos hidrossedimentológicos apresentam um grande potencial para serem utilizados no planejamento dos recursos hídricos, através desta ferramenta é possível estimar impactos causados pelas atividades antrópicas. Com base nessa premissa, utilizamos o modelo SWAT (Soil and Water Assessment Tools) na bacia rural denominada bacia Mutum Paraná, localizada nos municípios de Porto Velho e Nova Mamoré. Sua área $\left(771,2 \mathrm{~km}^{2}\right)$ tem influência direta na usina hidrelétrica de Jirau, e seu exutório localiza-se à montante da barragem. Os métodos aplicados foram o monitoramento hidrológico, geração de dados matriciais e tabulares, validação do modelo, análises estatísticas e avaliação espacial da produção do escoamento superficial e sedimento. O modelo SWAT gerou 27 subbacias com base nas características topográficas, mantendo como referência o rio principal. Os valores máximos de escoamento superficial e sedimento encontrados, respectivamente, são de $96,7 \mathrm{~mm} / \mathrm{ano}$ no e 3,35 ton/ha/ano, tendo sido obtido um $\mathrm{R}^{2}$ de 0,87 entre os valores de produção de sedimento e escoamento superficial simulados pelo modelo.

Palavras-chaves: Produção de sedimento. Modelagem hidrológica. Amazônia.

\section{APPLICATION OF THE SWAT MODEL AS A TOOL FOR HYDROSEDIMENTOLOGICAL ANALYZES IN THE HUTROGRAPHIC BASIN OF RIO MUTUM PARANÁ - RONDÔNIA}

Abstract: The use of hydrosedimentological models has great potential for use in water resources planning, through this tool and it is possible to estimate the impact caused by human activities. Based on this premise, we used the SWAT model (Soil and Water Assessment Tools) in the rural basin called the Mutum Paraná basin, located in the municipalities of Porto Velho and Nova Mamoré. Its area $\left(771.2 \mathrm{~km}^{2}\right)$ has a direct influence on the Jirau hydroelectric power plant, and its outlet is located upstream of the dam. The applied methods were hydrological monitoring, generation of matrix and tabular data, model validation, statistical analysis and spatial evaluation of the runoff and sediment production. The SWAT model generated 27 sub-basins based on topographic characteristics, maintaining the main river as a reference. The maximum values of runoff and sediment found, respectively, are $96.7 \mathrm{~mm} / \mathrm{year}$ in and 3.35 ton/ha/year, having obtained an $R^{2}$ of 0.87 between the values of sediment production and runoff simulated by the model.

Keywords: Sediment production. Hydrological modeling. Amazon.

\footnotetext{
${ }^{1}$ Universidade Federal de Rondônia, Departamento de Geografia, Porto Velho, Brasil, janyelson@gmail.com, https://orcid.org/0000-0003-1736-7741

2 Universidade Federal de Rondônia, Departamento de Geografia, Porto Velho, Brasil, dorisval@unir.br, https://orcid.org/0000-0003-4560-5444

${ }^{3}$ Universidade Federal de Rondônia, Departamento de Engenharia civil, Porto Velho, Brasil, tati.checchia@gmail.com, https://orcid.org/0000-0002-5772-4187
} 


\section{APLICACIÓN DEL MODELO SWAT COMO HERRAMIENTA DE ANÁLISIS HIDROSEDIMENTOLÓGICOS EN LA CUENCA HUTROGRÁFICA DE RIO MUTUM PARANÁ - RONDÔNIA}

Resumen: El uso de modelos hidrosedimentológicos tiene un gran potencial para ser utilizado en la planificación de los recursos hídricos, a través de esta herramienta es posible estimar los impactos causados por las actividades humanas. Con base en esta premisa, utilizamos el modelo SWAT (Suelo y Agua) en la cuenca rural denominada cuenca Mutum Paraná, ubicada en los municipios de Porto Velho y Nova Mamoré. Su área $\left(771,2 \mathrm{~km}^{2}\right)$ tiene una influencia directa sobre la central hidroeléctrica de Jirau, y su salida se encuentra aguas arriba de la presa. Los métodos aplicados fueron monitoreo hidrológico, generación de datos matriciales y tabulares, validación de modelos, análisis estadístico y evaluación espacial de la producción de escorrentías y sedimentos. El modelo SWAT generó 27 subcuencas en base a características topográficas, manteniendo el río principal como referencia. Los valores máximos de escorrentía y sedimento encontrados, respectivamente, son $96.7 \mathrm{~mm} /$ año en y 3.35 ton / ha / año, habiendo obtenido un $\mathrm{R}^{2}$ de 0.87 entre los valores de producción de sedimentos y escorrentía simulados por el modelo.

Palabras clave: Producción de sedimentos. Modelización hidrológica. Amazonas.

\section{Introdução}

Na Região Amazônica as comunidades e atividades econômicas têm fortes ligações com os recursos hídricos e se instalam em diversas áreas. Com a rápida expansão populacional nas últimas décadas, a demanda por recursos naturais aumentou significativamente, seja para a produção de alimento ou abastecimento público. Associado a este contexto há ainda o crescimento desordenado que, por vezes, é acompanhado de degradação ambiental.

Como consequências desse uso inadequado pode-se listar a queda da capacidade produtiva do solo, a degradação dos recursos hídricos, o favorecimento dos processos erosivos, que resultam em altas taxas de produção de sedimentos, bem como sua transferência para os recursos hídricos.

A produção de sedimentos pode ser entendida como a quantidade de partículas erodidas que são transportadas pela dinâmica hídrica da bacia hidrográfica. Tal produção está relacionada aos eventos naturais e antrópicos que, juntos, interagem e resultam numa maior ou menor produção de sedimentos.

Quando comparadas às regiões temperadas, as regiões tropicais e subtropicais apresentam uma maior ocorrência de eventos pluviométricos intensos, o que resulta no desequilíbrio do ciclo hidrossedimentológico, potencializando assim as suas consequências (TUCCI, 2005). A elevação da produção de sedimentos 
aumenta as chances de assoreamento de corpos hídricos e reduz a qualidade da água.

A modelagem da produção de sedimentos constitui-se em uma importante ferramenta para análise da distribuição espaço-temporal, fato que corrobora para a minimização dos custos altíssimos de monitoramento e possibilita a previsão de cenários ambientais alternativos.

Os modelos matemáticos de predição de erosão e de produção de sedimentos apresentam grandes potenciais para serem utilizados no planejamento com vista à sustentabilidade dos recursos naturais. Tendo como principais vantagens o tempo de resposta e o seu custo inferior ao custo de uma investigação experimental. Esse fator adquire maior importância à medida que o problema estudado apresenta maiores dimensões e complexidade, como uma bacia hidrográfica (PESSOA et al., 1997).

Levando-se em consideração as potencialidades da modelagem e a crescente expansão populacional da população amazônica nas últimas décadas, a demanda por recursos naturais tem aumentado significativamente, seja para a produção de alimento ou para o abastecimento público. Associado a este contexto há ainda o crescimento desordenado que, por vezes, é acompanhado de degradação ambiental.

É a partir dessas problemáticas que nasce este trabalho. O objetivo é avaliar a produção de água e sedimentos na Bacia hidrográfica do Mutum Paraná, localizada no estado de Rondônia, por meio do modelo SWAT, a fim de analisar as influências dos fatores fisiógrafos da área.

\section{Área de estudo}

A Bacia Hidrográfica Mutum Paraná (BHMP) localiza-se no noroeste do Estado de Rondônia, estendendo-se pelos municípios de Porto Velho e Nova Mamoré. O clima dessa região é caracterizado, de acordo com a classificação de Köppen (KOTTEK et al., 2006), como sendo Aw - clima tropical chuvoso, onde as temperaturas médias dos meses mais frios são superiores a $18^{\circ} \mathrm{C}$ (megatérmico), um período ecologicamente seco e sujeito a altos desvios pluviométricos ao longo dos anos (RONDÔNIA, 2002).

De acordo com os dados do Plano Agropecuário e Florestal de Rondônia, o PLANAFLORO (RONDÔNIA, 2002), a vegetação original encontrada na área da bacia é constituída por floresta ombrófila aberta, seguida por savana em áreas mais 
concentradas ao norte da bacia onde predomina a área plana. Dentro da savana, a fisionomia é de floresta com cipó, sendo maior parte aberta, pois a fitomassa e o fitovolume de recobrimento vão diminuindo gradativamente de densidade. Os tipos de solos da área de estudo são os latossolos vermelho - amarelos distróficos, que compreendem solos minerais profundos, com horizonte B latossólico e estrutura granular, apresentando textura média a muito argilosa e relevo plano. Abaixo podese observar na figura 1 a localização da área de estudo.

Figura 1 - Localização da área de estudo

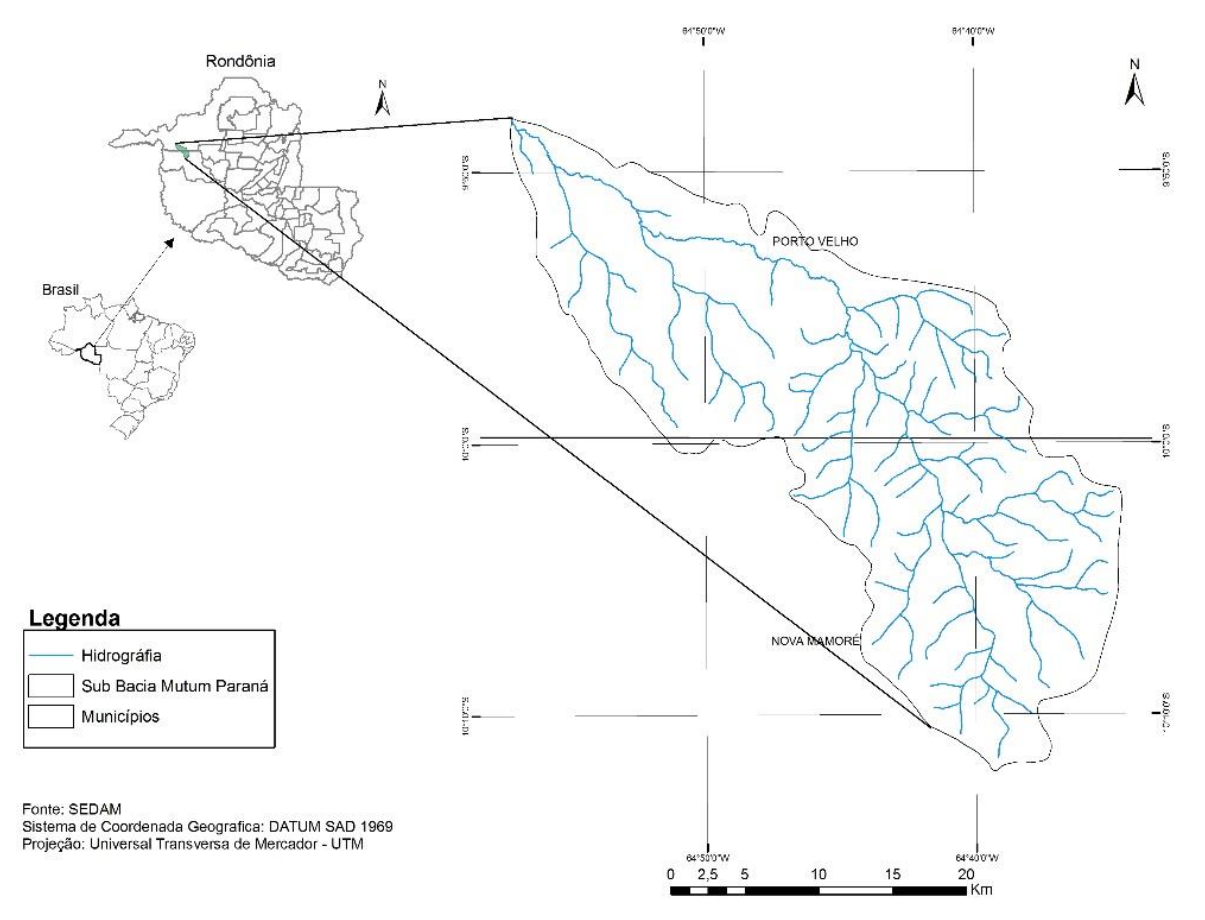

Fonte: Elaborado pelo autor, 2020.

As características geomorfológicas que constituem a BHMP são as planícies aluvionares constituídas por terrenos inundáveis e vales $e$ as unidades denudacionais constituídas pelas superfícies de aplainamento, com superfície de aplanamento inferior a 300m, dissecação média e nenhum ou esporádicos inselbergs e tors.

Os tipos de uso e cobertura encontrados na região são: floresta com 72,3\%, solo exposto com $0,9 \%$ e pastagem totalizando $26,8 \%$ da área total da bacia, sendo a principal atividade econômica da bacia e se encontrando em franca expansão, devido ao retorno rápido do capital investido e o baixo custo de investimento necessário para a criação de gado. 


\section{Material e métodos}

Base de dados espaciais

Dadas as características da área de estudo, foi construído um banco de dados contendo informações sobre a topografia, uso da terra, solos e hidrografia. Informações hidroclimáticas relativas à fluviometria, precipitação, temperatura, vento, umidade e radiação solar também integraram os dados que foram utilizados na alimentação do modelo para fins de calibração e de representação do comportamento hidrológico da bacia.

Foi utilizada a Estação Meteorológica de Jirau, gerenciada pela Secretaria de Estado do Desenvolvimento Ambiental (SEDAM) e localizada na área de influência da bacia. Esta estação forneceu os dados com a série histórica climática, constituída pelas variáveis: radiação solar $\left(\mathrm{m}^{2}\right)$; precipitação pluviométrica $(\mathrm{mm})$; temperaturas máxima, média e mínima $\left({ }^{\circ} \mathrm{C}\right)$; umidade relativa do ar $(\%)$ e velocidade do vento (m/s), no período de 2010 a 2012, com resolução temporal diária. As estações 01 , 02 e 03 utilizadas para monitoramento hidrológico foram instaladas e gerenciadas pela Universidade Federal de Rondônia (UNIR) com dados de precipitação e nível conforme pode ser observado na Figura 1, com área de $771,2 \mathrm{~km}$.

No levantamento e organização do banco de dados digital, foram utilizadas bases cartográficas do Sistema de Proteção da Amazônia (SIPAM) contendo as temáticas de vegetação, geomorfologia, pedologia PLANAFLORO (RONDÔNIA, 2002), bases cartográfica de Geologia do Serviço Geológico do Brasil (CPRM, 2007), cena de satélite Landsat-5/TM do ano de 2012 obtidas no site do INPE (2020) e cenas Advanced Spaceborne Thermal Emission and Reflection Radiometer (ASTER) Global Digital Elevation Model (GDEM) no site da NASA (USGS, 2019) para gerar os dados Topográficos da área de estudo.

- Base de Geológia - Serviço Geológico do Brasil - CPRM). Escala 1:250.000;

- Base de Geomorfológia - Governo do Estado de Rondônia - PLANAFLORO Escala 1:250.000;

- Base de Pedológica - Governo do Estado de Rondônia - PLANAFLORO Escala 1:250.000;

- Cena da Imagem Advanced Spaceborne Thermal Emission and Reflection Radiometer (ASTER) - (GDEM). Resolução espacial de 30m;

- Cena Imagens de Satélite Landsat-5 TM, bandas 3, 4 e 5, órbita/ponto 233/66 - INPE - 2011. Resolução espacial de 30m; 
- Dados Vetoriais de Limites Municipais e Estaduais - IBGE. Escala 1:100.000.

\section{Modelo numérico do terreno}

Para a elaboração do Modelo Digital de Elevação, foram utilizadas cenas do radar Advanced Spaceborne Thermal Emission Reflection Radiometer (ASTER) Global Digital Elevation Model (GDEM) referentes à área de estudo BHMP no software ARCGIS 9.3. Após a entrada deste dado utilizamos a Extension Contour, para gerar as curvas de nível com equidistâncias de $20 \mathrm{~m}$; em seguida criamos um shapefile na forma quadrática um pouco maior que a área de estudo para que não ocorresse o efeito de borda dentro da área de estudo; logo após fizemos o recorte das curvas geradas utilizando a Extension Clip e por fim geramos o MDE com a Extension $3 D$ analyst. Depois da obtenção desse procedimento, foi feito o préprocessamento dos dados digitais de elevação e drenagem.

\section{Dados de uso e cobertura da terra}

Para o levantamento de uso e cobertura da terra foram realizadas aplicações de formulários para definição das classes de uso e cobertura da terra e registro de ponto de coordenadas por meio de GPS, procedimento para a definição das classes. Para isso adotou-se o método do Instituto Brasileiro de Geografia e estatística (IBGE), que definiu o levantamento dos usos como "[...] o conjunto de operações necessárias à elaboração de uma pesquisa temática que pode ser sintetizada através de mapas" (IBGE, 2006). Neste trabalho, o uso e cobertura da terra será entendido como "[...] a distribuição geográfica da tipologia de uso, identificada através de padrões homogêneos da cobertura terrestre" (IBGE, 2006).

O mapa de uso e cobertura da terra foi obtido com técnicas de geoprocessamento e sensoriamento remoto a partir de base georreferenciada de GEOCOVER 2000 (USG, 2019) e imagens Landsat-05 bandas 5R,4G,3B. As imagens foram adquiridas junto ao Instituto Nacional de Pesquisas Espaciais (INPE, 2020) por meio de solicitação eletrônica e georreferenciadas utilizando a ferramenta Georeferencing. Os pontos amostrais para o georreferenciamento foram determinados através de identificação de pontos homólogos identificados em imagens e vetores já georreferenciados. 


\section{Dados pedológicos}

Os dados de solo necessário para a modelagem são características físicas e hídricas de cada classe de solo, o mapa de solos da BHMP com base no levantamento de solos (escala 1:250.000) realizado em 2002 pelo PLANAFLORO, bem como todos os dados tabulares exigidos pelo modelo. Os dados tabulares de solos necessários para o funcionamento do modelo SWAT são: grupos de saturação (HYDGRP), profundidade de raiz (SOL_ZMX), porosidade (ANION_EXCL), profundidade da camada (SOL_Z), densidade aparente (SOL_BD), capacidade de água no solo (SOL_AWC), condutividade hidráulica saturada (SOL_K), erodibilidade (USLE_K), albedo (SOL_ALB), carbono orgânico (SOL_CBN), e percentagem de argila, silte, areia. $\mathrm{Na}$ Tabela 1 podemos observar os dados tabulares de solos exigidos pelo modelo. 
Tabela 1 - Características físicas das classes de solo da bacia do rio Mutum Paraná Parâmetros Climáticos

\begin{tabular}{|c|c|c|c|c|c|c|c|c|}
\hline Dados & LAD & LEVD & LAD & LEVD & LAD & LEVD & LAD & LEVD \\
\hline NLAYERS & 1 & 1 & 2 & 2 & 3 & 3 & 4 & 4 \\
\hline HYDGRP & A & A & A & A & A & $A$ & $A$ & A \\
\hline SOL_ZMX & $>2200 \mathrm{~mm}$ & $>2200 \mathrm{~mm}$ & $>2200 \mathrm{~mm}$ & $>2200 \mathrm{~mm}$ & $>2200 \mathrm{~mm}$ & $>2200 \mathrm{~mm}$ & $>2200 \mathrm{~mm}$ & $>2200 \mathrm{~mm}$ \\
\hline SOL_Z & $150 \mathrm{~mm}$ & $100 \mathrm{~mm}$ & $550 \mathrm{~mm}$ & $400 \mathrm{~mm}$ & $900 \mathrm{~mm}$ & $800 \mathrm{~mm}$ & $>2200 \mathrm{~mm}$ & $>2200 \mathrm{~mm}$ \\
\hline SOLZ_BD & $1.25 \mathrm{~g} \mathrm{~cm}^{3}$ & $1.49 \mathrm{~g} \mathrm{~cm}^{3}$ & $2.5 \mathrm{~g} \mathrm{~cm}^{3}$ & $1.49 \mathrm{~g} \mathrm{~cm}^{3}$ & $1.25 \mathrm{~g} \mathrm{~cm}^{3}$ & $1.49 \mathrm{~g} \mathrm{~cm}^{3}$ & $1.25 \mathrm{~g} \mathrm{~cm}^{3}$ & $1.49 \mathrm{~g} \mathrm{~cm}^{3}$ \\
\hline SOL AWC & $0.5 \mathrm{~mm} / \mathrm{mm}$ & $0.5 \mathrm{~mm} / \mathrm{mm}$ & $0.5 \mathrm{~mm} / \mathrm{mm}$ & $0.5 \mathrm{~mm} / \mathrm{mm}$ & $0.5 \mathrm{~mm} / \mathrm{mm}$ & $0.5 \mathrm{~mm} / \mathrm{mm}$ & $0.5 \mathrm{~mm} / \mathrm{mm}$ & $0.5 \mathrm{~mm} / \mathrm{mm}$ \\
\hline SOL_CBN & $0.55 \%$ & $0.8 \%$ & $0.3 \%$ & $0.45 \%$ & $0.55 \%$ & $0.45 \%$ & $0.3 \%$ & $0.45 \%$ \\
\hline SOL K & $22.83 \mathrm{~mm} / \mathrm{hr}$ & $4.03 \mathrm{~mm} / \mathrm{kr}$ & $1.34 \mathrm{~mm} / \mathrm{hr}$ & $7.35 \mathrm{~mm} / \mathrm{hr}$ & $9.96 \mathrm{~mm} / \mathrm{hr}$ & $9.85 \mathrm{~mm} / \mathrm{hr}$ & $7.0 \mathrm{~mm} / \mathrm{hr}$ & $0.64 \mathrm{~mm} / \mathrm{hr}$ \\
\hline CLAY & $63 \%$ & $44 \%$ & $65 \%$ & $40 \%$ & $67 \%$ & $37 \%$ & $82 \%$ & $36 \%$ \\
\hline SILT & $10 \%$ & $7 \%$ & $10 \%$ & $7 \%$ & $12 \%$ & $7 \%$ & $7 \%$ & $7 \%$ \\
\hline SAND & $28 \%$ & $49 \%$ & $25 \%$ & $53 \%$ & $21 \%$ & $56 \%$ & $11 \%$ & $57 \%$ \\
\hline ROCK & $0 \%$ & 0 & $0 \%$ & $0 \%$ & $0 \%$ & $0 \%$ & $0 \%$ & $0 \%$ \\
\hline SOL ALB & 0.11 & 0.11 & 0.11 & 0.11 & 0.11 & 0.11 & 0.11 & 0.11 \\
\hline USLE $\mathrm{K}$ & 0.15 & 0.23 & 0.14 & 0.28 & 0.13 & 0.31 & 0.08 & 0.32 \\
\hline
\end{tabular}

LAD: Latossolo Vermelho Distrófico; LEVD: Latossolo Escuro Vermelho Distrófico; NLAYERS é o número da camada de informação (números de horizontes); HYDGRP é o grupo de mínima saturação hidráulica (A, B, C e D); SOL_ZMX é a profundidade máxima de solo que a raiz alcança $(\mathrm{mm})$; ANION_EXCL é a fração de porosidade do solo (fração); SOL_Z é a profundidade de cada horizonte $(\mathrm{mm})$; SOL BD é a densidade do solo $(\mathrm{g} \mathrm{cm}-1)$; SOL AWC é a capacidade de água do solo $(\mathrm{mm} \mathrm{mm}-1)$; SOL K é a condutividade hidráulica saturada (mm h-1); SOL_CBN é o conteúdo de carbono orgânico do solo (\% peso solo); CLAY é a porcentagem de argila no solo (\% peso solo); SILT é a porcentagem de silte no solo (\% peso solo); SAND é a porcentagem de areia no solo (\% peso solo); ROCK é a porcentagem de cascalho no solo (\% volume); SOL ALB é o albedo do solo (fração); e USLE K é o fator de erodibilidade do solo $(0,013$ ton $\mathrm{m} 2 \mathrm{hr}(\mathrm{m} 3-$ ton $\mathrm{cm})-1)$.

Fonte: Elaborado pelo autor, 2020 


\section{O modelo SWAT}

As simulações hidrológicas na bacia hidrográfica realizadas pelo modelo SWAT podem ser separadas em duas partes: fase terrestre e fase de propagação do ciclo hidrológico. A fase terrestre pode ser subdividida em sete componentes: hidrologia, clima, sedimentos, crescimento vegetal, manejo agrícola, nutrientes e pesticidas. Já a fase de propagação do ciclo hidrológico é dividida em dois componentes: propagação do canal principal e propagação no reservatório. Como o intuito deste estudo foi simular a hidrossedimentalogia da BHMP, abordou-se apenas os componentes hidrologia e sedimentos da fase terrestre.

\section{Hidrologia}

O componente hidrologia é a base para a simulação da produção de água superficial, sendo sua modelagem hidrológica regida pela equação do balanço hídrico (Arnold et al. 1998; Neitsch et al. 2002; Bonumá et al. 2009; Castro, 2013), representada pela equação 1 abaixo:

$$
S W_{t}=S W+\sum_{t=1}^{t}\left(R_{t}-Q_{i}-E T_{i}-P_{i}-Q R_{i}\right)
$$

Onde: Swt é a quantidade final de água no solo $(\mathrm{mm})$; SW0 é a quantidade inicial de água no solo $(\mathrm{mm})$; Ri é o total diário precipitado $(\mathrm{mm})$; Qsi é o total diário de escoamento superficial $(\mathrm{mm})$; ETi é o total diário de evapotranspiração $(\mathrm{mm})$; wi é o total diário percolado $(\mathrm{mm})$ e Qgwi é a vazão de retorno diária $(\mathrm{mm})$.

Segundo Neitsch et al. (2002), o modelo utiliza uma formulação modificada do Método da Curva Número (CN), do Soil Conservation Service (SCS, 1972), para calcular o escoamento superficial da classe, uso e manejo do solo, equação 2 abaixo.

$$
Q_{\text {surf }}=\frac{\left(\boldsymbol{P}_{i}-\boldsymbol{I}_{a}\right)^{2}}{\left(\boldsymbol{P}_{i}-\boldsymbol{I}_{a}+S\right)}
$$

Onde: Qsurf é o escoamento superficial total $(\mathrm{mm})$; Pi é a precipitação total $(\mathrm{mm})$; la é a abstração inicial (o armazenamento no terreno, a interceptação e a infiltração no solo antes de iniciar o escoamento superficial em $\mathrm{mm}$ ) e $\mathrm{S}$ é o parâmetro de retenção $(\mathrm{mm})$ o qual varia com o uso, podemos calcular de acordo com a equação 3. 


$$
S=25,4 \cdot \frac{100}{C N}-10
$$

Onde: $C N$ (número de deflúvio) é um valor adimensional que variar entre 1 (cobertura muito permeável) e 100 (cobertura completamente impermeável), dependendo da umidade, tipos e usos do solo.

O modelo utiliza o Método Racional Modificado para calcular a vazão de pico (Neitsch et al., 2002), abaixo podemos observar a equação utilizada.

$$
Q_{\text {pico }}=\frac{C N . i A}{3,6}
$$

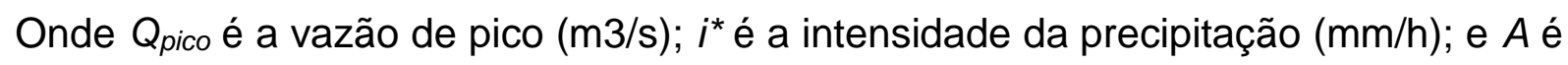
a área da bacia $\left(\mathrm{km}^{2}\right)$, e 3,6 é um fator de conversão de unidades.

\section{Sedimentos}

A produção de sedimentos da BHMP foi estimada por meio da Equação Universal de Perda de Solo Modificada (MUSLE - Modified Universal Soil Loss Equation). Esta equação, segundo Lino (2009), é a versão modificada da Equação Universal de Perda de Solos (USLE - Universal Soil Loss Equation) desenvolvida por Wischmeier e Smith (1978). Ela tem como objetivo simular a erosão bruta e a produção de sedimentos a partir do escoamento superficial, representando assim a energia utilizada na desagregação e transporte de sedimentos. Segundo os autores, a modificação na equação trouxe alguns benefícios, como eliminação da taxa de transporte, uso em eventos isolados e maior precisão no modelo (WISCHMEIER; SMITH, 1978).

A Equação Universal de Perda de Solo Modificada utilizada pelo modelo SWAT é apresentada pela equação 5, a seguir:

$$
\text { Sed }=11,8 \cdot\left(Q_{\text {surf }} \cdot q_{\text {peak } 8} a_{r e a}\right)^{0,56} \cdot K_{U S L E} \cdot C_{U S L E} \cdot P_{U S L E} \cdot L S_{U S L E} \cdot C F R G
$$

Onde: sed é a produção de sedimentos na bacia (t/dia); $Q_{\text {surf é o escoamento }}$ superficial para a bacia $(\mathrm{mm} / \mathrm{ha}) ; q_{\text {peak }}$ é a vazão de pico $\left(\mathrm{m}^{3} / \mathrm{s}\right)$; areahru é a área da bacia (ha); KusLE é o fator de erodibilidade do solo; CUSLE é o fator de cobertura e manejo do solo (adimensional); PUSLE é o fator de práticas conservacionistas (adimensional), LSUSLE é o fator topográfico (comprimento e inclinação da rampa) e CFRG é o fator de fragmento grosseiro (rochas). 
O fator $\mathrm{K}$ de Erodibilidade do solo representa a suscetibilidade do solo em ser erodido, e é dado pelas características intrínsecas do mesmo. Wischmeier e Smith (1978) desenvolveram a seguinte equação (6) para calcular o fator erodibilidade do solo:

$$
K_{\text {usle }}=\frac{0,00021-M^{1,14} \cdot(12-O M)+3,25-\left(c_{\text {soillstr }}-2\right)+2,25 \cdot\left(c_{\text {perm }}-3\right)}{100}
$$

Onde: $K$ é o fator de erodibilidade do solo; $M$ é o parâmetro de tamanho das

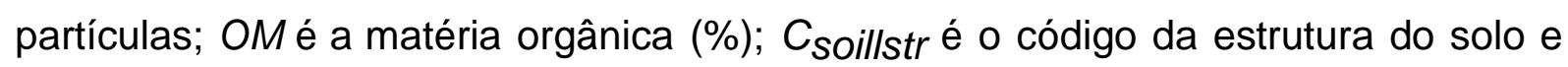

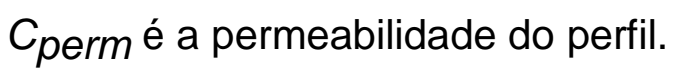

O código da estrutura do solo e a permeabilidade do perfil são características tabeladas presentes no banco de dados do modelo SWAT. O fator de cobertura e manejo do solo é definido segundo Lino (2009, p. 150) como a taxa de perda de solo durante o ciclo de crescimento da planta, sendo determinado pela equação 7 a seguir:

$$
C_{\text {usle }}=\exp \left(\left[\operatorname{in}(0,8)-i n\left(C_{\text {usle,mn }}\right)\right] * \exp \left[-0,00115 * r s d_{\text {surf }}\right]+i n\left[C_{U S L E}\right]\right)
$$

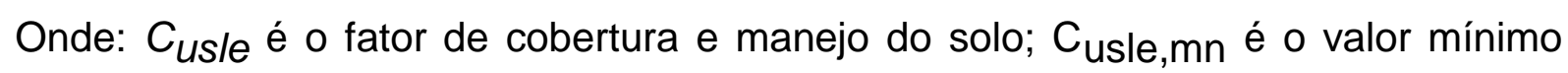

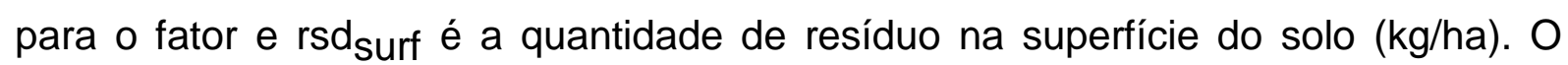
valor mínimo do fator de cobertura é obtido pela equação 8, apresentada abaixo

$$
C_{\text {usle }}=1,463 i n\left[C_{U S L E}\right]+0,1034
$$

Segundo Wischmeier e Smith, (1978, p. 69), o fator de práticas conservacionistas expressa a relação entre a intensidade de perdas de solo quando uma prática conservacionista é empregada e quando a cultura está plantada no sentido do declive.

O fator comprimento e inclinação da rampa (fator topografia), segundo Marchioro (2008, p. 196), é calculado pelo modelo hidrológico SWAT utilizando a equação 9, abaixo:

$$
L S_{\text {usle }}=\left(\frac{L_{\text {hill }}}{22,1}\right)^{m} *\left(65,41 * \operatorname{sen}^{2}+\left(a_{\text {hill }}\right)+4,56 * \operatorname{sena}_{\text {hill }}+0,065\right)
$$

Onde: Lhill é o comprimento da encosta $(\mathrm{mm}) ; m$ é o fator exponencial e $\boldsymbol{\alpha}_{\text {hill }}$ é o ângulo da declividade da encosta $\left(^{\circ}\right)$. 


\section{Calibração do modelo}

Segundo os autores Bravo et al. (2007) e Shawul et al. (2013), a calibração consiste em uma técnica de otimização dos parâmetros do modelo hidrológico, buscando adequar os dados simulados com os dados reais. Esta técnica pode ser realizada de forma manual, pelo próprio usuário, ou automática, utilizando softwares complementares.

Conforme Marchioro (2008), para a calibração do modelo SWATé necessário um banco de dados climático, vazão e concentração de sólidos em suspensão monitorados por um período de no mínimo 5 a 10 anos, visando assim minimizar os erros durante a calibração. Arnold et al. (1998) complementa afirmando que caso não haja informações de qualidade disponíveis para a calibração, deve-se dispensar esta etapa, pois esta não é requerida pelo modelo para realizar a simulação.

Segundo apontam os estudos de Uzeika (2009) e Castro (2013), o modelo SWAT sem calibração apresentou um bom desempenho na estimativa do escoamento superficial, vazões líquidas e evapotranspiração.

Sendo assim, para realizar a simulação hidrossedimentológica da Bacia Hidrográfica Mutum Paraná foi dispensada a calibração do modelo, devido a bacia não ser instrumentada e por falta de dados monitorados de vazão pelo período mínimo requerido.

\section{Resultados e discussão}

O material disponível para transporte existente nas vertentes das bacias tem sua origem na erosão causada pelo impacto da gota da chuva sobre o solo exposto e na calha dos rios, surgindo da erosão das margens, do fundo e da descarga de abastecimento de montante. Assim, maiores índices de pluviosidade são proporcionais a maiores índices de produção de sedimento; enquanto que menores índices de pluviosidade resultam em menores índices de produção de sedimento.

Na Figura 2, abaixo, podemos observar o gráfico de precipitação média anual para os anos de 2012 a 2013. O ano de 2012 é considerado climatologicamente típico, considerando os totais mensais precipitados. O ano de 2013 teve uma tendência normal durante o primeiro semestre, seguindo no segundo semestre, referente ao início do ano hidrológico de 2014, com volumes precipitados acima da média. O resultado do volume extra foi a cheia histórica de 2014, cuja o período de recorrência superou os 150 anos. 
Figura 2 - Gráfico de precipitação média anual dos anos de 2012 a 2013

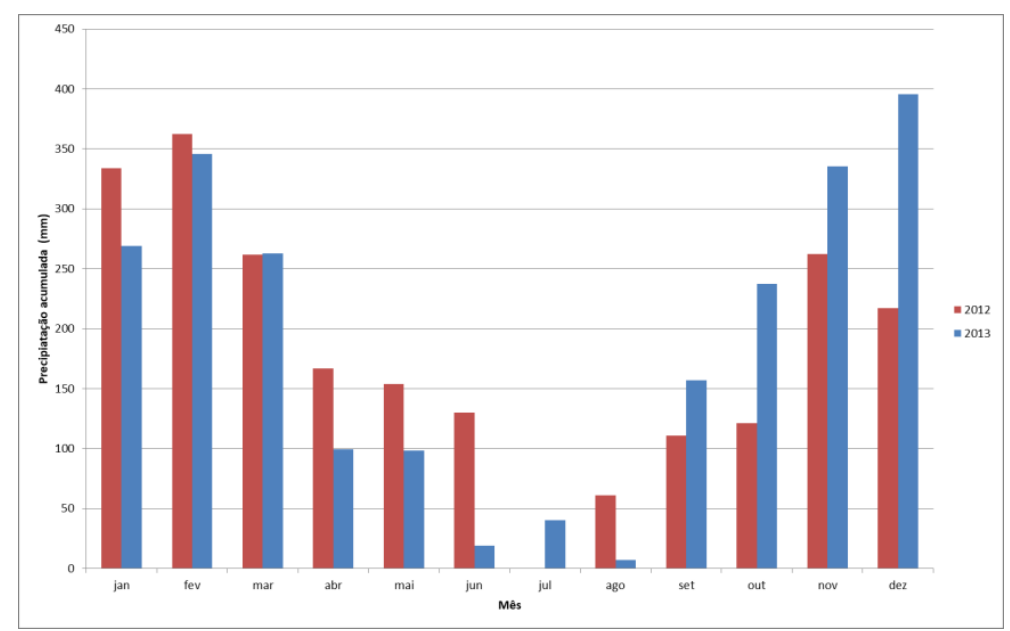

Fonte: Elaborado pelo autor, 2020.

Os índices de precipitação apresentaram bons resultados tanto quanto a consistência e correlação dos processos simulados, os valores de $\mathrm{R}^{2}$ da precipitação simulada e observada foram de 0,78 e 0,87 para os anos de 2012 e 2013, respectivamente. Este resultado mostra uma boa sensibilidade à sazonalidade das chuvas na região, tendo em vista que, analisando o período a partir do início do ano hidrológico (mês de setembro), o ano de 2013 teve volume precipitado 58\% superior a 2012.

O modelo SWAT subdividiu a BHMP em 27 sub-bacias com base nas características de elevação do terreno, mantendo como referência a ramificação principal do corpo d'água, conforme a Figura 3 a seguir. De acordo com Arnold et al. (2011) a subdivisão da bacia em sub-bacias garante a distribuição espacial dos parâmetros de declividade, uso e cobertura da terra e tipos de solo, preservando a homogeneidades das características da bacia. 
Figura 3 - Distribuição espacial do escoamento superficial das sub-bacias do Rio Mutum Paraná para o ano de 2012 e 2013

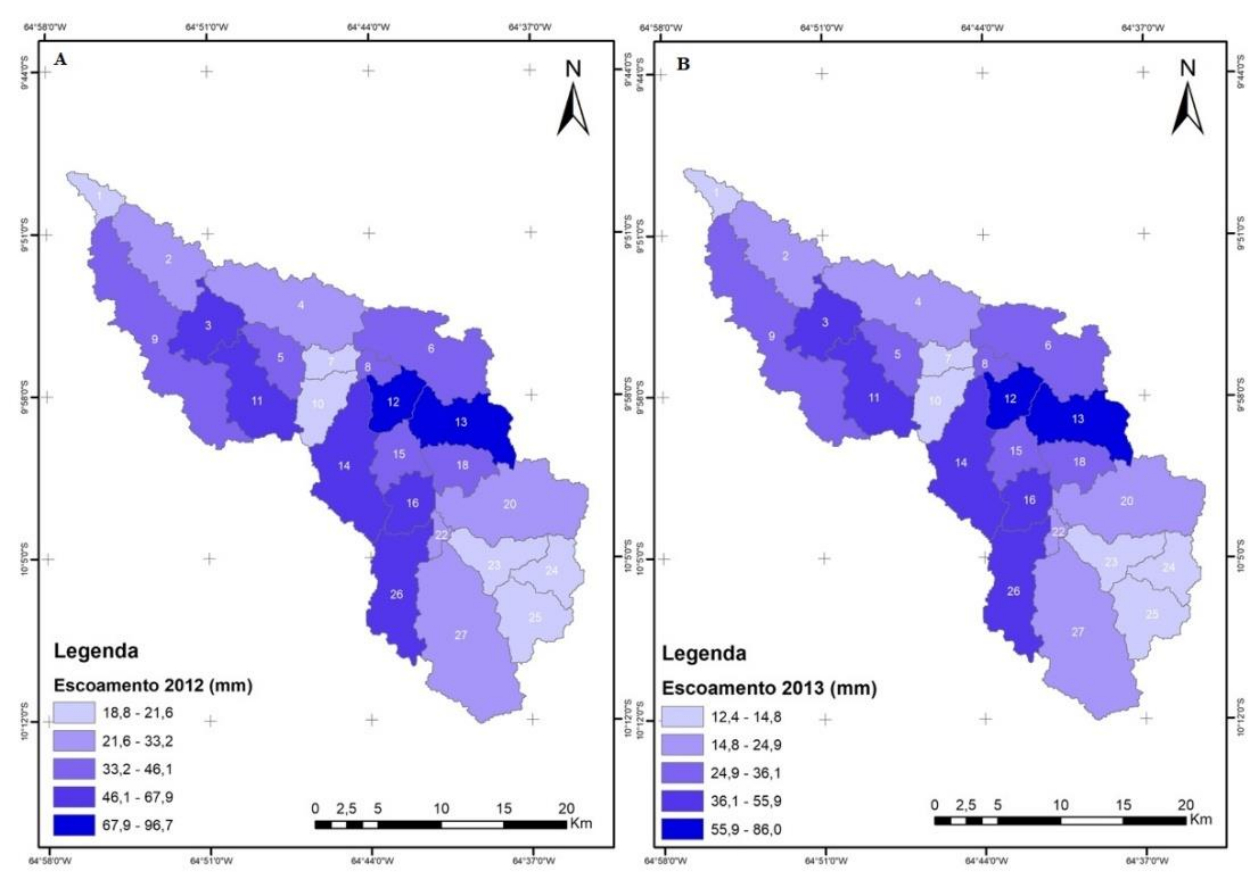

Fonte: Elaborado pelo autor, 2020.

O escoamento superficial simulado resultou em uma variação de $18,8 \mathrm{~mm}$ a 96,7 mm no ano de 2012, já para o ano de 2013 os resultados foram de $12 \mathrm{~mm}$ a 86 $\mathrm{mm}$. Os maiores valores estão concentrados na sub-bacia 12 e 13 variando de 91,5 $\mathrm{mm}$ e $96,1 \mathrm{~mm}$ respectivamente. Esse valor é explicado pelas suas características pedológicas e de uso da terra, uma vez que se detecta a presença de latossolos que contribuem significativamente para o aumento do escoamento superficial associada a presença de solo exposto. Segundo Ruthes et al. (2012) solos com textura argilosa ou muito argilosa, como os Latossolos, tendem a uma maior agregação das partículas causando selagem da cobertura do solo, o que diminui a capacidade de infiltração e consequentemente aumenta o escoamento superficial.

As sub-bacias 3, 11, 14, 16 e 26 apresentam valores médios de escoamento superficial, os valores encontrados variaram entre $51,8 \mathrm{~mm}$ e $67,9 \mathrm{~mm}$. Nessas áreas foram observados que o uso do solo predominante foi o pasto, tendo 0 pisoteio dos animais resultando na compactação do solo, dificultando a infiltração de água no perfil.

As demais sub-bacias apresentaram valores menores quanto ao escoamento superficial, variando entre $18 \mathrm{~mm}$ a $21 \mathrm{~mm}$. Nestas áreas a cobertura do solo predominante é de floresta ombrófila aberta caracterizada naturalmente por uma camada espessa de serapilheira que reduz a velocidade do escoamento, aumentando a capacidade de infiltração da água no solo. 
Paralelo ao escoamento superficial tem-se a produção de sedimentos na bacia. Para as sub-bacias 1, 2, 4, 7 e 10, localizadas em região mais plana da bacia e cujo uso do solo predominante é a floresta, a produção de sedimentos variou entre 0,119 t/ha/ano e 0,358 t/ha/ano para o ano de 2012 e 0,0004 t/ha/ano a 0,188 t/ha/ano para o ano de 2013. Os valores mais elevados de produção de sedimentos foram encontrados nas sub-bacias 12, 13 e 16, variando de 2,029 t/ha/ano a 3,441 t/ha/ano para 2012 e 1,298 t/ha/ano a 2,357 t/ha/ano para 2013. As sub-bacias 12 e 13 são caracterizadas por apresentar solo exposto. Sem a cobertura vegetal de proteção o sedimento é deslocado de acordo com a energia disponibilizada durante o processo. A sub-bacia 16 possui mais de $70 \%$ da área composta por pecuária, sendo o pisoteio dos animais a principal componente que potencializa a produção de sedimentos na bacia.

As sub-bacias localizadas na região sul da BHMP possuem características topográficas com declividades mais acentuadas. Associadas a predominância de florestas nativas, a produção de sedimentos permanece baixa. Desta forma a produção de sedimentos fica entre 0,358 t/ha/ano e 3,441 t/ha/ano para 2012 e $0,187 \mathrm{t} / \mathrm{ha} /$ ano e 2,357 t/ha/ano para 2013. Outra característica que potencializa o processo de sedimento nestas áreas é o fato de estarem em áreas intermediarias como encontro de estradas ou confluências de canais, gerando, assim, valores acentuados de produção de sedimento.

Nas sub-bacias onde predominam áreas de floresta ombrófila natural, obtivemos os menores valores de produção de sedimento. Um dos fatores que contribui para essa baixa produção de sedimento é a declividade da área, classificada como plana na maior parte das sub-bacias. Como consequência, o escoamento superficial encontrado também foi baixíssimo, variando entre $18 \mathrm{~mm}$ a $36 \mathrm{~mm}$. Na Figura 4 podemos observar os valores da distribuição espacial da produção de sedimento analisadas anteriormente. 
Figura 4 - Distribuição espacial da produção de sedimento das sub-bacias do Rio Mutum Paraná para o ano de 2012 e 2013
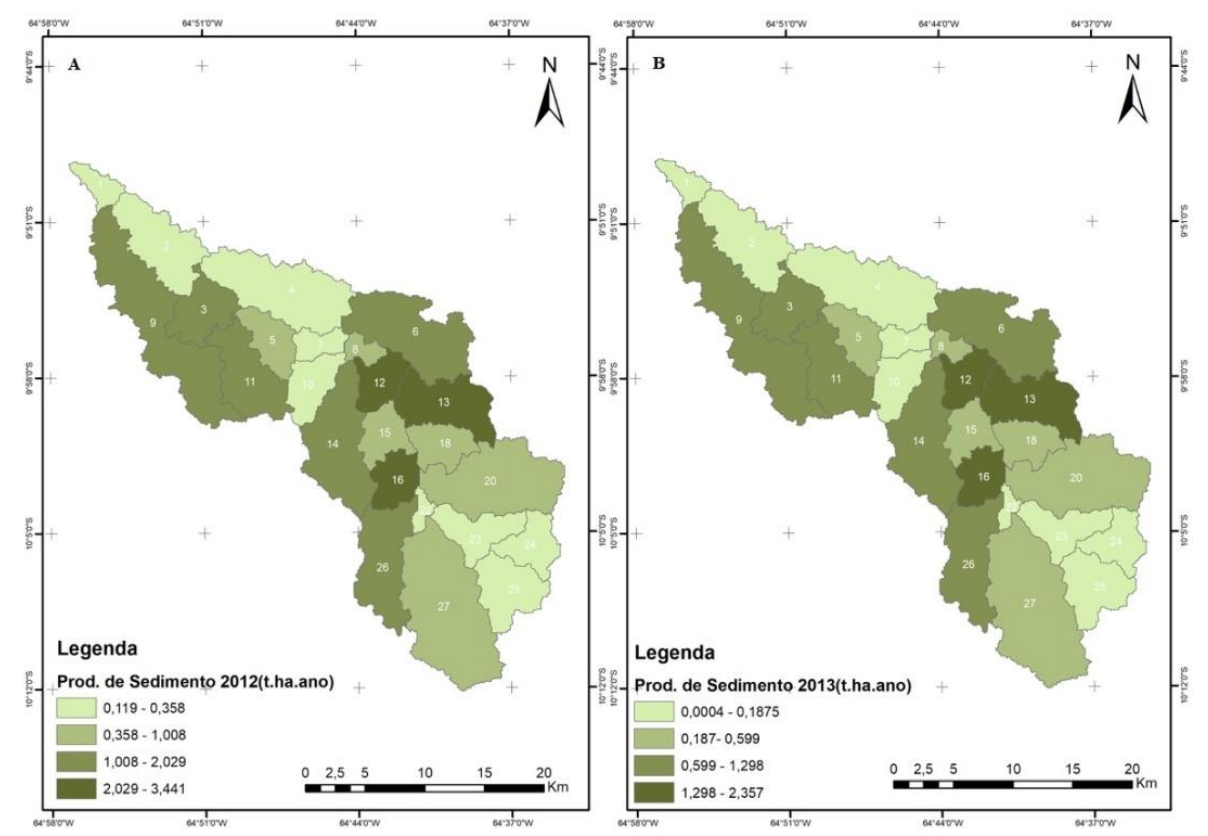

Fonte: Elaborado pelo autor, 2020.

Analisando a Figura 5 constata-se que a produção de sedimento e o escoamento superficial simulados tiveram uma boa correlação estatística, com $R^{2}$ de 0,87 . Utilizando uma equação linear como base para os cálculos, os dados correlacionados tiveram um bom ajuste linear acompanhando os picos de chuva e vazão do período, já citados anteriormente.

Figura 5 - Gráficos de correlação estatísticas do escoamento e produção de sedimento

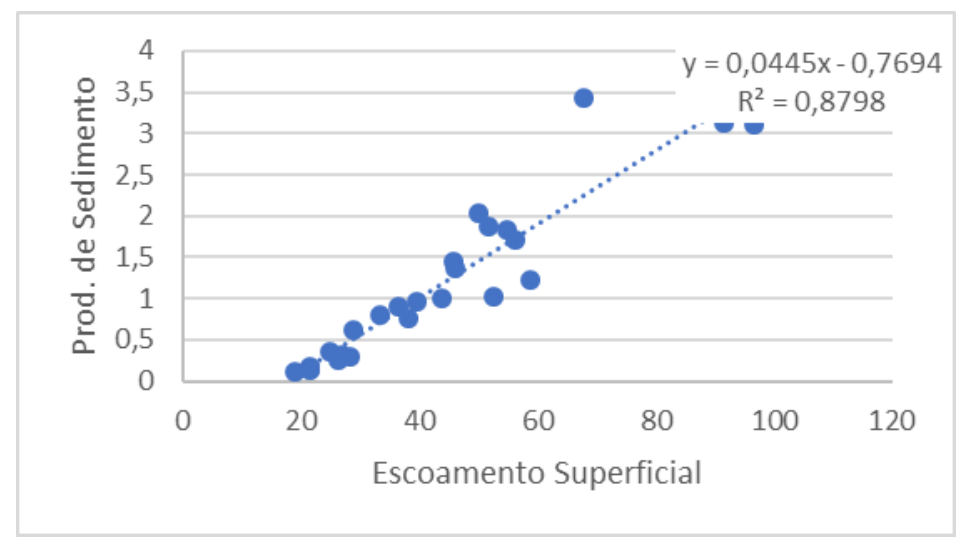

Fonte: Elaborado pelo autor, 2020.

Os valores de vazão observados em campo, juntamente com o escoamento superficial simulado pelo SWAT, se assemelham este modelo com algumas ressalvas. No mês de novembro, dezembro e fevereiro os valores de escoamento obtidos excederam os valores de vazão. Parte desse problema se deve ao fato de 
que apenas parte do escoamento superficial chega rapidamente aos cursos d'água para contribuir na vazão. Acreditamos que nos meses em que o modelo superestimou o escoamento superficial, isso ocorreu devido à limitação do modelo em simular a água disponível no solo durante os períodos chuva. Cabe salientar que os valores de vazão observados em campo foram insuficientes para calibrar o modelo adequadamente.

O modelo SWAT apresentou resposta satisfatória quanto a esse processo de simulação da produção de sedimento, considerando as proporcionalidades dos outros parâmetros analisados, como precipitação e a produção de agua observadas.

\section{Considerações Finais}

A utilização de modelos hidrossedimentológicos como ferramenta para o planejamento na região da Amazônia é extremamente promissor. Rondônia possui um banco de dados fisiográficos capaz de alimentar modelos que permitem planejar a região de forma adequada, evitando decisões que comprometam a qualidade ambiental local.

A forma de uso e cobertura da terra e a declividade do terreno possuem influência direta nos processos hidrossedimentológicos da Bacia Hidrográfica Mutum Paraná, sendo que as sub-bacias ocupadas, em sua grande maioria, por florestas apresentaram valores menores de produção de sedimento, variando de 0,11 ton/ha.ano a 0,90 ton/ha.ano; e as sub-bacias em que o uso e cobertura da terra é do tipo solo exposto ou pastagem a produção foi elevada, entre 2,029 ton/ha.ano e 3,441 ton/ha.ano. A topografia do terreno também apresentou influência sobre a produção de sedimento, sendo encontrados nas sub-bacias mais planas os menores valores de perdas de solo.

As interações entre as características desses dois fatores influenciam na produção de escoamento superficial, o qual está diretamente relacionado com a produção de sedimentos. Esses resultados nos mostram que o modelo apresenta uma boa sensibilidade à alteração de uso, cobertura da terra e a topografia do ambiente.

A medida de controle a ser aplicada nestas bacias a fim de frear a perda de solo nesses ambientes está em estabelecer e manter as áreas de preservação permanente ao longo dos cursos d'água. É uma medida que acorda com a Lei $n$. 4.771 de 1965 (BRASIL, 1965) e com o Plano Nacional de Recursos Hídricos (MMA, 2006), que recomenda relacionar a agricultura com a aptidão, mercado e 
sustentabilidade ambiental, evitando-se a degradação das condições sociais e ambientais. Outra recomendação possível é aprofundar a orientação sobre as práticas de conservação de vegetação, solos e água. A abertura de novas vias de acesso deve ser planejada a fim de não potencializar a produção de sedimentos na bacia.

Os resultados obtidos ainda ressaltam a necessidade de reconhecer não somente a proteção nas áreas de proteção permanente, mas também as áreas nas bacias hidrográficas sensíveis aos processos erosivos e que necessitam de práticas de controle de erosão ou mudanças de uso e cobertura da terra.

\section{Referências Bibliográficas}

ARNOLD, J. G.; SRINIVASAN, R.; MUTTIAH, R. S. \& WILLIAMS, J. R. Large area hydrologic modeling and assessment - part $\mathbf{i}$ : model development. Journal of the American Water Resources Association, v. 34, n. 1, p. 73-89, fev. 1998.

ARNOLD, J. G.; KINITY, J. R.; SRINIVASAN, R.; WILLIAMS, J.R.; HANEY, E.B. \& NEITSCH, S. L. SWAT input/output file documentation version 2009. Texas, Grassland Soil and Water Research Laboratory, 2011. 643p.

BONUMÁ, N. B.; REICHERT, J. M.; MINELLA, J. P.; JACOMET, L.; OLIVEIRA, A. \& RODRIGUES, M.F. Simulação da produção de sedimentos em uma pequena bacia hidrográfica rural no sul do brasil. In: CONGRESSO BRASILEIRO DE CIÊNCIA DO SOLO, n. 32, Fortaleza, 2009.

BORTOLOTTO, N. L. Avaliação hidrossedimentológica da bacia do rio cunha, município de rio dos cedros, santa catarina com o modelo swat. Trabalho de Conclusão de Curso. (Graduação em Engenharia Sanitária e Ambiental) - Universidade Federal de Santa Catarina, Santa Cataria, 2011.101 p.

BRASIL. Lei Federal $\mathbf{n}^{\circ} \mathbf{4 . 7 7 1}$, de 15 de setembro de 1965. Estabelece o Código Florestal Brasileiro. Diário Oficial da República Federativa do Brasil. Brasília, DF. 16 set. 1965.

Ministério do Meio Ambiente. Plano nacional de recursos hídricos: síntese executiva. Brasília: MMA, 2006.

BRAVO, J.M.; PICCILLI, D. G. A.; COLISCHONN, W.; TASSI, R.; MELLER, A. \& TUCCI, C. E. M. Avaliação visual e numérica da calibração do modelo hidrológico iph ii com fins educacionais. In: SIMPÓSIO BRASILEIRO DE RECURSOS HÍDRICOS, n. 17. São Paulo, $\mathrm{ABRH}, 2007$.

CARVALHO, N. de O. Hidrossedimentologia prática. Rio de Janeiro: CPRM, 1994. 372 p.

CASTRO, K.B. Avaliação do modelo swat na simulação da vazão em bacia agrícola do cerrado intensamente monitorada. Dissertação (Mestrado em Geociências) Universidade de Brasília, Brasília, 2013. 122 p.

CONSERVATION SERVICE SOIL - SCS. Hydrology. In: National engineering handbook. Washington: USDA, 1972. p.101-1023.

EMPRESA BRASILEIRA DE PESQUISA AGROPECUÁRIA - EMBRAPA. Súmula da 10. Reunião Técnica de Levantamento de Solos. Rio de Janeiro: SNLCS, 1979. 83 p. 
INSTITUTO BRASILEIRO DE GEOGRAFIA E ESTATÍSTICA - IBGE. Manual técnico de uso da terra/ ibge, coordenação de estudos naturais e ambientais. 2. ed. Rio de Janeiro: IBGE, 2006.

INSTITUTO NACIONAL DE PESQUISAS ESPACIAIS - INPE. Catálogo de imagens. 2020. Disponível em: <http://www.dgi.inpe.br/CDSR/>. Acesso em: 13 mar. 2020.

KOTTEK M., GRIESER J., BECK C., RUDOLF B., RUBEL F., 2006: World Map of the Köppen-Geiger climate classification updated. Meteorologische Zeitschrift. 15, 259-263. 2006. Disponível em: <http://koeppen-geiger.vu-wien.ac.at/pdf/kottek_et_al_2006_A4.pdf>. Acesso em: 24 mai. 2020.

KUHNLE, R.A.; BINGER, R.L.; FOSTER, G.R. \& GRISSINGER, E.H. Effect of land use changes on sediment transport. Water Resources Research, 32. p. 3189-3196, 1996.

LINO, João Félix de Luca. Análise da dinâmica hidrossedimentológica da bacia hidrográfica do rio preto (sc) com o modelo swat. Dissertação (Mestrado em Engenharia Ambiental) - Universidade Federal de Santa Catarina, Florianópolis, 2009. 150 p.

MARCHIORO, E. Modelagem hidrossedimentológica na bacia do córrego santa maria: subsídios à aplicação de práticas de conservação de água e solo no noroeste fluminense. Tese (Doutorado) Programa de Pós-graduação em Geografia - Universidade Federal do Rio de Janeiro, 2008. $196 \mathrm{f}$.

NEITSCH, S. L.; ARNOLD J. G.; KINIRY J. R.; WILLIAMS J. R. Soil and water assessment tool. Theoretical Documentation, Version 2005. Temple, Texas: Blackland Research Center, Texas Agricultural Experiment Station, 2005.

PESSOA, M. C. P. Y.; LUCHIARI, A. J.; FERnANDES, E. N. \& LIMA, M. A. Principais modelos matemáticos e simuladores utilizados para análise de impactos ambientais das atividades agrícolas. Jaguariúna, EMBRAPA/CNPMA, 1997. 83 p.

RODRIGUES, L. C.; BOVO-SCOMPARIN, V. M.; TRAIN, S.; SUSICLEY, J.; BORTOLINI, J. C.; PAULA, A. C. M.; REIS, L. M. Grupos morfológicos funcionais da planicie de inundação do alto rio Paraná. In: XIV CONGRESSO BRASILEIRO DE FICOLOGIA, 2012. João Pessoa: SBFIC, 2012.

RONDÔNIA. Plano Agropecuário e florestal de rondônia - planafloro. Porto Velho: TECNOSSOLOS, Relatório Técnico, 2002.

RUTHES, J.M.; TOMAZONI, J.C. \& GOMES, T.C. Propriedades do solo da bacia hidrográfica do rio catorze que contribuem no processo erosivo laminar. In: SEMINÁRIO DE INICIAÇÃO CIENTÍFICA E TECNOLÓGICA DA UTFPR, 17, Anais do SICITE 2012. Curitiba: Editora da UTFPR, 2012.

SERVIÇO GEOLÓGICO DO BRASIL - CPRM, 2007. Mapa geológico e dos recursos minerais do estado de rondônia. 2007. Disponível em: <http://www.cprm.gov.br/publique/media/geologia_basica/cartografia_regional/mapa_rondoni a.pdf>. Acesso em: 15 mar. 2019.

SHAWUL, A.A.; ALAMIREW, T.; DINKA, M.O. Calibration and validation of swat model and estimation of water balance components of shaya mountainous watershed, southeastern ethiopia. Hydrology and Earth System Sciences, 2013. p.13955-13978.

TUCCI, C. E. M. Modelos hidrológicos. 2. ed. Porto Alegre: Editora da UFRGS, 2005. p. 104.

UNITED STATE GEOLOGICAL SERVEY - USGS. Earth Explorer. Disponível em: <http://earthexplorer.usgs.gov/>. Acesso em: 12 jan. 2019.

UZEIKA, T. Aplicabilidade do modelo swat (soil and water assessment tool) na simulação da produção de sedimentos em uma pequena bacia hidrográfica rural. 
Programa de Pós-graduação em Recursos Hidráulicos e Saneamento Ambiental. Dissertação (Mestrado em Recursos hídricos e Saneamento Ambiental) - Universidade Federal do Rio Grande do Sul, Porto Alegre, 2009. $132 \mathrm{f.}$

WISCHMEIER, W.H.; SMITH, D.D. Predicting rainfall erosion losses: a guide to conservation planning. Agriculture Handbook $N^{\circ}$ 537. Washington, U.S. Government Printing Office, 1978. $69 \mathrm{p}$.

\section{NOTAS DE AUTOR}

\section{CONTRIBUIÇÃO DE AUTORIA}

Janielson da Silva Lima - resultado de uma dissertação de mestrado, Concepção. Coleta de dados, Análise de dados, Elaboração do manuscrito, revisão e aprovação da versão final do trabalho

Dorisvalder Dias Nunes -Participação ativa da discussão dos resultados; orientação; Revisão e aprovação da versão final do trabalho.

Tatiane Emílio Checchia - Participação ativa da discussão dos resultados; Coorientação; Revisão e aprovação da versão final do trabalho.

\section{FINANCIAMENTO}

Bolsa de Pesquisa nível mestrado - Universidade Feral de Rondônia - Unir/CNPq

Apoio logístico, ecobatimetro, estações pluviométricas - FINEP Ct-Hidro 01/2010. Processo n. 004998.10

\section{CONSENTIMENTO DE USO DE IMAGEM}

Não se aplica.

\section{APROVAÇÃO DE COMITÊ DE ÉTICA EM PESQUISA}

Não se aplica.

\section{CONFLITO DE INTERESSES}

Não se aplica.

\section{LICENÇA DE USO}

Este artigo está licenciado sob a Licença Creative Commons CC-BY. Com essa licença você pode compartilhar, adaptar, criar para qualquer fim, desde que atribua a autoria da obra.

\section{HISTÓRICO}

Recebido em: 11-07-2019

Aprovado em: 08-08-2020 